\title{
FABRICATION OF A HOLLOW BULB OBTURATOR FOR A HEMI- MAXILLECTOMY DEFECT: A CASE REPORT
}

Mukesh Yadav¹, Vandana Singh², Pooja Hazari³ ${ }^{3}$ Divya Pandey ${ }^{4}$, Shreyans Damade ${ }^{5}$

\section{HOW TO CITE THIS ARTICLE:}

Mukesh Yadav, Vandana Singh, Pooja Hazari, Divya Pandey, Shreyans Damade. "Fabrication of a Hollow Bulb Obturator for a Hemi- Maxillectomy Defect: A Case Report". Journal of Evolution of Medical and Dental Sciences 2014; Vol. 3, Issue 22, June 02; Page: 6147-6151, D0I: 10.14260/jemds/2014/2715

ABSTRACT: Benign or malignant tumors of mouth and oropharyngeal region often result in the maxillofacial defects. The acquired maxillectomy defects results in the impairment of speech, mastication, swallowing and facial esthetics. The Prosthodontist plays a significant role in the rehabilitation of the acquired maxillectomy defects with the special emphasis to the reduction of the weight of the prosthesis and thereby enhancing the retention and stability. The weight of the obturator can cause a dislodging force. It must therefore be as light as possible. A suitable technique of fabrication should be selected to achieve this. This case report describes the fabrication of a hollow bulb obturator for a hemi- maxillectomy patient (Aramany's class 1 defect) who had undergone wide excision and alveolar resection squeal of management of adenoid cystic carcinoma of the right palate. This article describes the step by step clinical and laboratory procedure involved in the rehabilitation of a hemimaxillectoy dentate patients using obturator lined with soft liner to restore the functions such as esthetics, mastication, deglutition and speech.

KEYWORDS: Hemimaxillectomy, Maxillofacial prosthesis, Definitive hollow bulb obturator.

INTRODUCTION: Maxillectomy is treatment option for maxillary cancer that leaves the patient with a palatal defect, which may cause problem with swallowing, mastication and speech. These functional problems and changes in appearances may result in psychological problem.1,2

The defect frequently is complex and involves the skin, bone, muscle, cartilage and multilayer of mucosa. Therefore reconstruction of such defect is often challenging. To rehabilitate such patient require multidisciplinary approach.

Palatal defects those are treated prosthodontically seal congenital or acquired defects and contiguous structures. Obturators close or seal these defects allowing the restoration to fulfill esthetics and functions like mastication, deglutition and speech. ${ }^{3}$

Materials and techniques used for fabrication of this obturator vary from patient to patient depending upon location and extent of defect. Prosthetic rehabilitation not only restores esthetics and functions but also boosts patient's moral. This case report describes the clinical and laboratory procedures for the patient of right partial maxillectomy with a definitive hollow bulb obturator.

Clinical Case Report: A moderately built 36 years old male patient reported to the Department of Prosthodontics, People's College of Dental Sciences \& Research Centre, Bhopal (M.P.) with a chief complaint of difficulties in taking food and speech and leakage of food and liquid from the oral cavity into the nasophaynx and restricted mouth opening. An obvious nasal twang was observed in the speech of the patient. The lower arch was completely dentulous. 
His past medical history revealed that he had under went right inferior partial Maxillectomy (from right central incisor to 3rd molar as a treatment for adenoid cystic carcinoma of one month back).

On examination, the right side of his face was found to be slightly depressed inwards and gave an unaesthetic appearance. The patient had a large defect extending from left incisor region to the soft palate. Palatal grafting was done to minimize the defect. The defect was Aramany class 1 but was compromised by the absence of entire quadrant of teeth. The defect was unilateral and bounded medially by the mid line of the hard palate, laterally by the buccal mucosa, and posteriorly by the soft palate (figure 1).

Treatment plan included fabrication of a hollow bulb obturator with cast partial denture frame work replacing the entire right quadrant of teeth.

\section{Step by Step Treatment \& Lab Procedure:}

- Patient reported to the department with restricted mouth opening of $17 \mathrm{~mm}$. In order to increase the mouth opening Trismus screw appliance was advised to the patient for one month. After one month $38 \mathrm{~mm}$ of mouth opening was recorded (figure 2).

- Then preliminary upper and lower impressions were made with irreversible hydrocolloid (alginate) using the perforated stock tray, taking care to block out defect undercuts with petrolatum laden gauge and a stone cast was poured from the impression to obtain a diagnostic cast. An interim prosthesis was fabricated to facilitate healing of the lesion.

- Three months later defect was completely healed and then mouth preparation was done and rest seats were prepared on left second premolar and first molar, to receive embrasure clasps. Guide plane was prepared on left central incisor.

- Final impression was made with putty wash technique using silicone putty impression material (figure 3). The impression was boxed and poured with type IV dental stone to obtain final master cast. The defect was outlined on the master cast and surveyed for designing the metal frame work.

- Master cast was blocked out by block out wax.

- The master cast was duplicated with the help of reversible hydrocolloid impression material (agar-agar) and invested by investing material and a refractory cast was obtained. A frame work was waxed on to the refractory cast. The wax pattern was cast in $\mathrm{Co}-\mathrm{Cr}$ alloy and the metal frame work was obtained.

- To reduce the weight of the prosthesis, a hollow bulb was fabricated with lost salt technique. It was made with autopolymerising acrylic resin. A layer of autopolymerising acrylic resin was adapted onto the defect area, which was filled with salt and again a layer of autopolymerising acrylic resin was adapted to cover the defect. After the complete polymerization, a hole was made with a round bur and bulb was washed with the help of water syringe so as to remove the salt from the bulb. The hole was closed with autopolymerising resin only after removing the water and salt completely.

- The occlusal rims were fabricated over the frame work to record the jaw relation and articulated on the semiadjustable articulator (Hanau). After teeth arrangement, try in of waxed up denture was performed in the conventional manner, and the patient was satisfied with the 
appearance and speech. After verification of the jaw relation, the trial denture was processed in conventional manner for final cast partial prosthesis fabrication.

- Soft liner was applied on the intaglio surface of hollow bulb in order to provide extra comfort, better retention and stability to the prosthesis.

- The final prosthesis was inserted into the patient mouth and it was checked for proper palatal contour and peripheral seal (figure 4,5 ). The patient was educated about the maintenance of the prosthesis.

- The patient had showed remarkable improvement in taking food and drinking liquids without any nasal regurgitation. And his speech was much improved.

- Post insertion check-up was done at one week, one month, and three month intervals and the patient was very much satisfied with the treatment.

DISCUSSION: The present case is a classical Aramany's class I defect which is unilateral; the entire palate on right side was resected along the midline. ${ }^{4}$ And the teeth on left side were left intact.

The design can be either linear or tripodal, two or three anterior teeth are splinted whenever possible, and support is derived from the central incisor and the most posterior abutment tooth. If the dental arch is curved the principle of effective indirect retention is utilized by the location of the rest on the canine or on the distal surface of the 1st premolar in a tripodal design. Direct retention is obtained either from the labial surface of the anterior teeth with I bar on the central incisor.

The obturator should be kept simple, light weight and inexpensive. The weight of the obturator is reduced, making it more comfortable and efficient. Also, the lightness of the obturator improves one of the fundamental problems of retention and increases physiological function so that teeth and supporting tissue is not stressed unnessecerily ${ }^{5,6}$

CONCLUSION: Though it is difficult to improve the quality of life for hemimaxillectomy patients compared with patients with conventional prostheses, this can be achieved with skill, knowledge and experience of specialists. The problem experienced by hemimaxillectomy patients are reduced if a team approach is adopted and specialists are careful to apply skill and experience at all stages and keep the patient under regular review.5,7 The fabrication of an acrylic hollow bulb obturator it's challenging, if conventional flasks cannot be used or when special maxillo-facial flasks are not available. 5,8

Finally, when the patient received and inserted the obturator, his aesthetic appearance and self-confidence improved dramatically.

\section{REFERENCES:}

1. Ackerman AJ. The prosthetic management of oral and facial defects following cancer surgery. J Prosthet Dent 1955; 5: 413-38.

2. Desjardins RP. Early rehabilitative management of the maxillectomy patient. J Prosthet Dent 1977; 38:311-8.

3. John Beumer III, Thomas, A. Curtis, David N Firtell. Restoration of acquired hard palate defects, C. V. Mosby, 1979, 188-205.

4. Mohamed A Aramany. Basic principles of obturator design for partially edentulous patients. Part I: Classification. J Prosthet Dent 2001; 86 (6): 559-561. 


\section{CASE REPORT}

5. Ramninder Kaur Bawa, Kamleshwar Kaur, Simrat Kaur, Imandeep Singh. Prosthodontic management of dentate hemimaxillectomy patient: a case report. Indian Journal of Comprehensive Dental Care 2011:1(1); 75-77.

6. Des Jardius RP. Obturator prosthesis design for acquired maxillectomy defects. J Prosthet Dent 1978; 39:424-35.

7. F Keyf. Obturator prostheses for hemimaxillectomy patients. Journal of Oral Rehabil 2001; 28: 821-829.

8. K E Brown. Fabrication of a hollow-bulb obturator. J Prosthet Dent 1969; 21: 97.

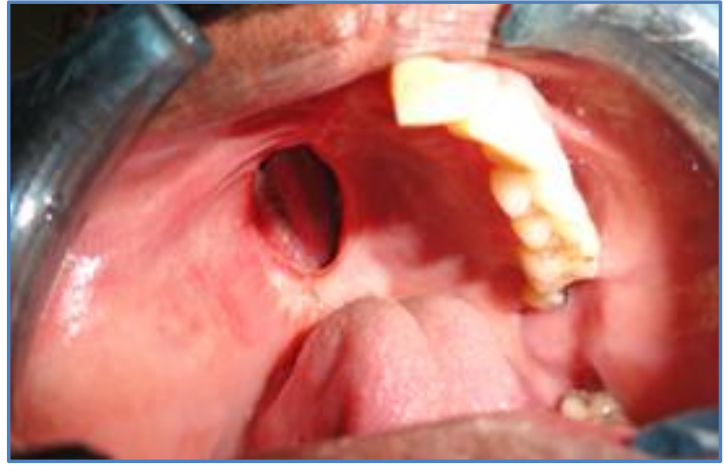

Fig. 1: Intra-oral defect on right side

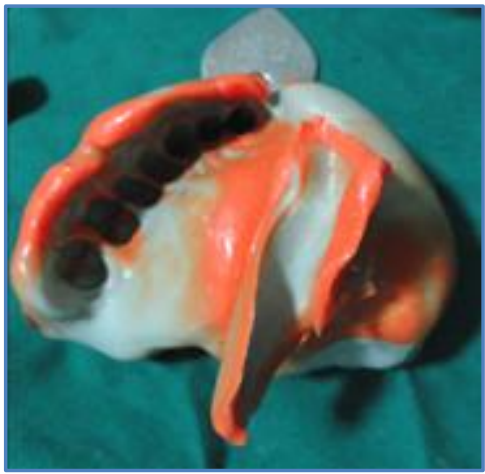

Fig. 3: Final impression

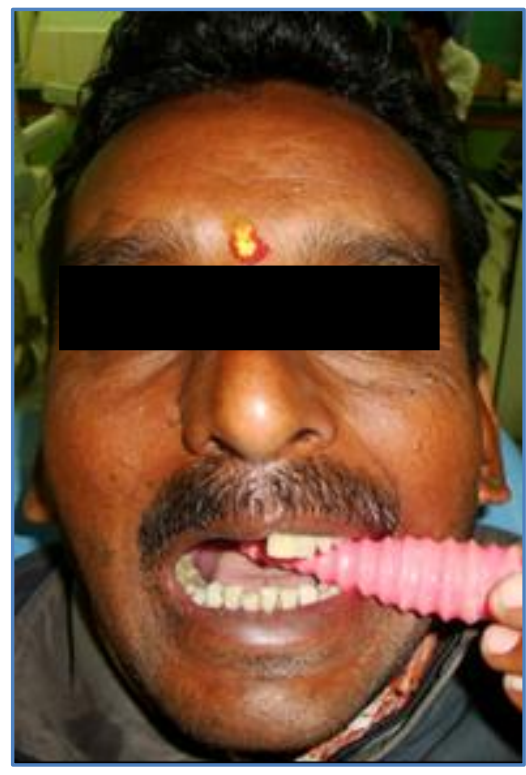

Fig. 2: Restricted Mouth Opening

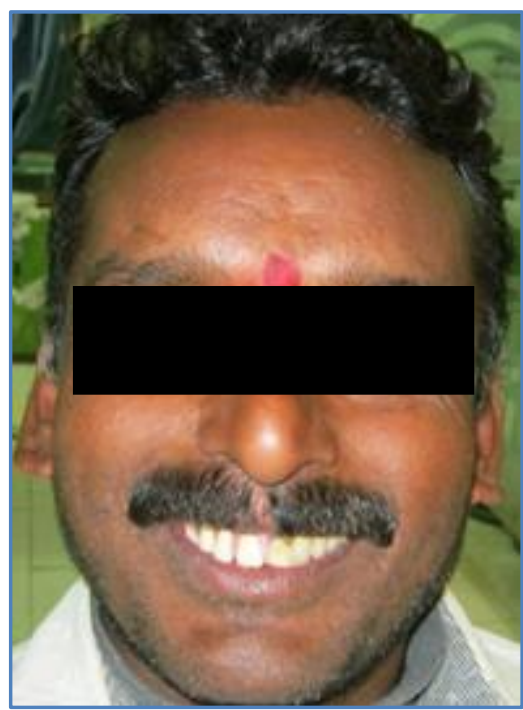

Fig. 4: Postprosthetic frontal view 


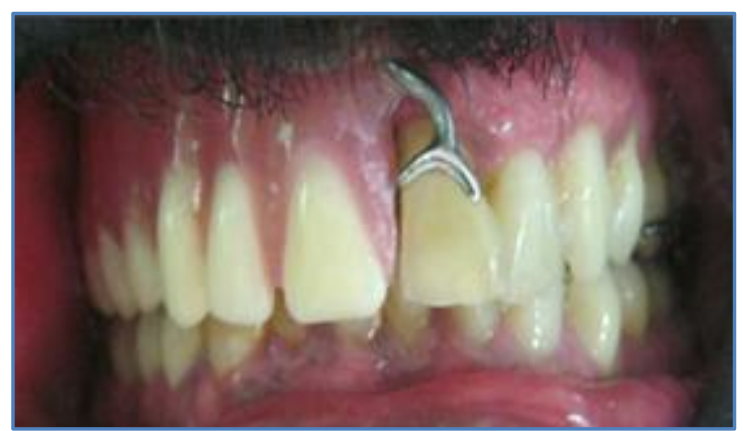

Fig. 5: Intraoral placement of prosthesis in occlusion

\section{AUTHORS:}

1. Mukesh Yadav

2. Vandana Singh

3. Pooja Hazari

4. Divya Pandey

5. Shreyans Damade

\section{PARTICULARS OF CONTRIBUTORS:}

1. Senior Lecturer, Department of Prosthodontics, Guru Gobind Singh College of Dental Sciences \& Research Centre, Burhanpur (M.P.)

2. Senior Lecturer, Department of Oral Medicine \& Radiology, Bhabha College of Dental Sciences, Bhopal (M.P.)

3. Senior Lecturer, Department of Prosthodontics, RKDF Dental College \& Research Centre, Bhopal (M.P.)

4. Senior Lecturer, Department of Conservative \& Endodontics, Bhabha College of Dental Sciences, Bhopal (M.P.)
5. Post Graduate Student, Department of Prosthodontics, People's College of Dental Sciences \& Research Centre, Bhopal (M.P.)

\section{NAME ADDRESS EMAIL ID OF THE} CORRESPONDING AUTHOR:

Dr. Mukesh Yadav, C/o Dr. Vandana Singh, I-31 Sant Asharam Nagar,

Bagsevania, Bhopal (M.P.) - 462043

Email: mdsmukeshyadav@gmail.com

Date of Submission: 17/05/2014. Date of Peer Review: 19/05/2014. Date of Acceptance: 26/05/2014. Date of Publishing: 31/05/2014. 